\title{
Estimation of the centre of mass from motion capture and force plate recordings: A study on the elderly
}

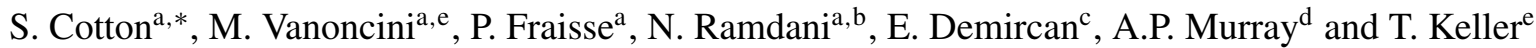 \\ ${ }^{a}$ Department of Robotic, LIRMM, Université de Montpellier 2, Montpellier, France \\ ${ }^{\mathrm{b}}$ CERTES, Université Paris Est - Créteil Val de Marne, France \\ ${ }^{\mathrm{c}}$ Department of Mechanical Engineering, Stanford University, Stanford, CA, USA \\ ${ }^{\mathrm{d}}$ Department of Mechanical Engineering, Dayton University, Dayton, OH, USA \\ ${ }^{\mathrm{e}}$ Health and Quality of Life Unit, Fatronik-Tecnalia, Donostia, San Sebastian, Spain
}

\begin{abstract}
The estimation of the centre of mass position in humans is usually based on biomechanical models developed from anthropometric tables. This method can potentially introduce errors in studies involving elderly people, since the ageing process is typically associated with a modification of the distribution of the body mass. In this paper, an alternative technique is proposed, and evaluated with an experimental study on 9 elderly volunteers. The technique is based on a virtual chain, identified from experimental data and locating the subject's centre of mass. Its configuration defines the location of the centre of mass, and is a function of the anatomical joint angles measured on the subject. This method is a valuable investigation tool in the field of geronto-technology, since it overcomes some of the problems encountered with other CoM estimation methods.
\end{abstract}

Keywords: Centre of mass, estimation, human, motion capture, force plate, elderly

\section{Abbreviations}

CоM
CoP
SESC
$\mathrm{T}_{\mathrm{i}}$

$\mathbf{A}_{\mathbf{i}}$

$\mathbf{A}_{\mathbf{i}}^{*}$

$\mathrm{D}^{+}$

$d_{i}$

$c_{i}$
Centre of Mass

Centre of Pressure

Statically Equivalent Serial Chain

homogeneous transform matrix between

link $i-1$ and link $i$

rotation matrix between link $i-1$ and link $i$

aggregation of rotation matrices $\mathbf{A}_{\mathbf{i}}$

aggregation of matrices $\mathbf{A}_{\mathbf{i}}^{*}$

pseudo-inverse of the matrix $D$

translation vector between link $i-1$ and link $i$

position of the CoM of the $i^{t h}$ link expressed

in its reference frame

\begin{tabular}{|c|c|}
\hline$n_{i}$ & mass of the ith segment of the body \\
\hline$M$ & total mass of the body \\
\hline$\theta_{i}$ & anatomical joint angles (ankle, knee, hip) \\
\hline$r_{i}$ & length of the ith segment of the SESC \\
\hline $\mathbf{R}$ & vector of the parameters $\boldsymbol{r}_{i}$ of the SESC \\
\hline$n$ & $\begin{array}{l}\text { number of degrees of freedom of the model } \\
\text { and of the SESC }\end{array}$ \\
\hline$m$ & $\begin{array}{l}\text { number of static poses used for the identification } \\
\text { of the SESC }\end{array}$ \\
\hline $\operatorname{CoM}_{x}, \operatorname{CoM}_{y}$ & $\begin{array}{l}\text { coordinates of the projection of the CoM on } \\
\text { the ground }\end{array}$ \\
\hline$F_{x}, F_{y}, F_{z}$ & forces measured by the force platforms \\
\hline $\begin{array}{l}M_{x}, M_{y}, M_{z} \\
\mathrm{RMS}\end{array}$ & $\begin{array}{l}\text { moments measured by the force platforms } \\
\text { Root Mean Square }\end{array}$ \\
\hline
\end{tabular}

\footnotetext{
*Corresponding author. E-mail: cotton@lirmm.fr.
} 


\section{Introduction}

The ability to maintain balance in upright standing is a basic requirement during daily activities. It is a pre-requisite for independent living, but can be challenging for elderly people due to inevitable transformations associated with ageing: increase in reaction times, deterioration of visual and sensory feedback, and modifications of the biomechanical properties of the muscles [9, 14, 23-25]. Poor balance capabilities can result in falls in the elderly, possibly requiring hospitalization, surgical intervention and rehabilitation. Research has also highlighted long term psychological consequences: the fear of falling can lead to a deterioration of the quality of life, as the individual limits the range of activities that he or she feels safe to autonomously perform $[11,17]$. Understanding the mechanisms underlying human balance, and their modifications due to the ageing process, is of fundamental importance in research aimed at improving the quality of life by increasing independence in later life. In fact, such information could guide the development of assistive devices, fall prevention tests and rehabilitation procedures.

The study of balance capabilities in humans is often conducted by considering the variation of the Centre of Pressure $(\mathrm{CoP})$ or the Centre of Mass (CoM) positions during a movement of interest [3, 18], or in response to a chosen perturbation $[12,21]$. The CoP can be directly computed from force platform measurements, but can only provide limited information since its position is defined in a two dimensional space corresponding to the the plane of the platforms [16]. The CoM can be estimated from the joint angles, measured with electrogoniometers or a motion capture system, provided that the parameters (i.e. the mass and CoM position) for each segment of the body are known. Typically, this information is obtained from anthropometric tables, notably those published by Zatiorsky, Winter, Dempster and De Leva $[7,8,26,27]$. These works have been of fundamental importance in the field of biomechanics, since they provided data which are essential for any study of the dynamics of human gesture. Moreover, they allowed for the variations in the body parameters due to differences in body-build. However, they only account for variations within a relatively small category of subjects (e.g. healthy young adult Caucasians as in Zatsiorsky and Seluyanov [27]). Several authors have highlighted this limitation, and tried to improve the anthropometric tables to make them more realistic, for example, for infants [19] or specific ethnic groups [22].

The use of anthropometric tables is particularly problematic when dealing with individuals characterized by an atypical distribution of the body mass; the higher the deviation from the "nominal" distribution, the higher the modelling error. For example, Galli et al. proposed the introduction of corrective factors to take into account such deviations, and applied this concept in an investigation on the obese population [10]. The estimation of these corrective factors, however, remains difficult to validate. Other authors employed more complex (and computationally expensive) models, based on Finite Elements Modelling techniques (e.g. the study on injury risk during pregnancy [15]). Researchers working in the field of Functional Electrical Stimulation in paraplegia faced the same difficulties while trying to develop appropriate biomechanical models for the paraplegic population. In fact, the changes induced by a spinal cord injury (lack of mobility, changes in the metabolism, etc.) usually lead to an increase in the mass of the upper part of the body (because of fat accumulation or muscle hypertrophy) and a decrease of the mass lower limbs due to atrophy.

With the goal of predicting the CoM location of an articulated system of bodies, especially while the system is moving, the literature contains several methods dedicated to this task. The most common approach is to estimate the horizontal location of the CoM by recording the CoP, generally using data generated via a force platform, and then using this information in manipulations of Newtons equations. Following Shimbas work [20], King and Zatiorsky have proposed a method utilizing the double integration of the horizontal ground reaction forces [13]. The challenge posed by this method is the determination of the initial constants of integration, a difficult problem in light of force platform sampling rates. Breniere et al. detail the relationship between the CoM and the $\mathrm{CoP}$ in the frequency domain, but it is a relationship best suited to addressing periodic motions $[1,2]$. These methods produce acceptable CoM estimation error but remain restricted to horizontal CoM estimation and constrain the subject to stay on the force plates. These methods are consequently not usable in our study where the aim is to produce spatial estimation of the centre of mass.

In previous studies $[5,6]$ a new method, producing a spatial estimation of the CoM for humanoid 
robots and humans, was presented and validated. The method, based on the concept of the Statically Equivalent Serial Chain, or SESC, requires the acquisition of force platform data and joint angles during an initial set-up phase, in which the subject under study is asked to maintain a certain number of static postures. The data collected during this phase are used to find the parameters of the SESC which defines the position of the CoM. Once the SESC is defined, the position of the CoM only depends on the configuration of the SESC, which is a function of the joint angles measured on the human subject. This means that the position of the CoM can be estimated from the measured values of the joint angles, but without incurring in the potential limitations of a biomechanical model developed using anthropometric tables.

The advantages offered by the new technique make it particularly suited for studies aimed at improving the quality of life for elderly people for two main reasons. The first is related to the changes in the body-build due to ageing, as elderly and young subjects differ both in the body mass index (BMI) and the mass distribution. Hence, any method relying on anthropometric tables will introduce unknown errors in the estimation of the CoM position, which are likely not to be negligible. The second reason is that the method proposed here only requires the measurement of joint angles to provide a single, compact variable (the CoM position) suitable for a study on balance capabilities. Such measurements can be obtained from a motion capture system, but also from portable, minimally invasive devices such as electrogoniometers and gyroscopes. The only other information required consist in the measurement of the ground reaction forces and moments, but these readings are only necessary for the initial set-up phase. Therefore, this method will allow investigations considering experimental data collected in a home environment, and during activities of daily living.

This paper is structured as follows: firstly, a review of the SESC modeling technique and its experimental identification is presented considering a general three dimensional case. Subsequently, the method is applied to the elderly population: a simplified model, appropriate for the specific study considered, is described Finally, the estimated of the CoM are discussed in the light of a comparison with the results which would be obtained if a method based on anthropometric tables were to be used.

\section{Estimation process}

This section contains a review of the notations, equations, and previous results that will prove useful to a discussion about the estimation of the centre of mass of a human subject.

\subsection{Statically equivalent serial chain modelling}

A simple example will be used to show how a mechanical system with a tree or chain structure can be transformed into an equivalent serial chain, locating its centre of mass. The same concepts will be applied in section 3, to transform the anthropometric structure of an elderly subject to a serial chain locating the subject's centre of mass, see Fig. 1.

\subsubsection{Kinematic and static parameters}

The systems under study are assumed to be composed of rigid bodies, called links, connected by revolute or spherical joints. As such, each link is fully described by its geometric and mass properties. Thus, for each link, the mass and the location of the centre of mass are known, as are the locations of all joints. Homogeneous transforms, denoted $\mathbf{T}_{\mathbf{i}}$, are used to relate the reference frames attached to any two bodies in the system,

$$
\mathbf{T}_{\mathbf{i}}=\left[\begin{array}{cc}
\mathbf{A}_{\mathbf{i}} & \boldsymbol{d}_{i} \\
\mathbf{0} & 1
\end{array}\right]
$$

where $\mathbf{A}_{\mathbf{i}}$ is a 3 -by-3 rotation matrix, $\boldsymbol{d}_{\boldsymbol{i}}$ is a 3-by-1 displacement vector, and the $\mathbf{0}$ represents a $1-b y-3$ vector of zeros. A 3-by-1 vector $\boldsymbol{c}_{\boldsymbol{i}}$ is used to locate the $\mathrm{CoM}$ of an individual body in the local reference frame attached to body $i$, or relative to $\mathbf{T}_{\mathbf{i}}$. Finally, the mass of body $\mathrm{i}$ is given by $m_{i}$ where the total mass of the system is $M=\sum m_{i}$.

\subsubsection{SESC modelling}

A brief review of the main steps in the development of the statically equivalent serial chain of the example chain depicted in Fig. 2(a) is now presented. The centre of mass of any multi-link chain, $\boldsymbol{C o M}$, with a serial or a branched chain structure, can be expressed as the end-effector of a SESC. Figure 2(b) illustrates this point for the branched chain depicted in Fig. 2(a). The process begins with the definition of the centre of mass of a collection of bodies, or the weighted sum of each body's centre of mass location, Equation (2). 


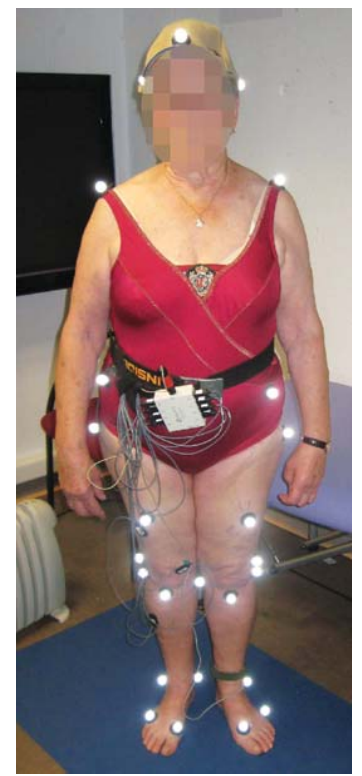

(a) Subject

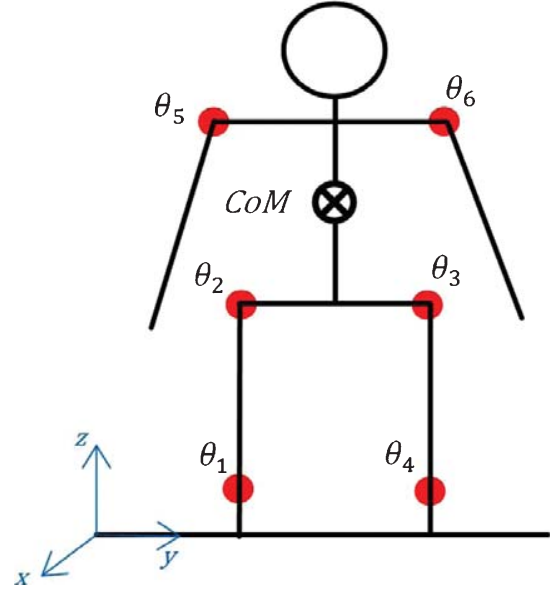

(b) Subject's Kinematic model

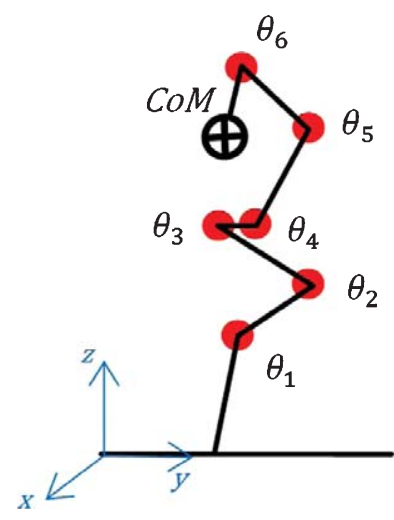

(c) Subject's SESC

Fig. 1. These pictures illustrate the transformation process going from the subject (a) to her kinematic model (b) and finally her SESC (c) locating her CoM.

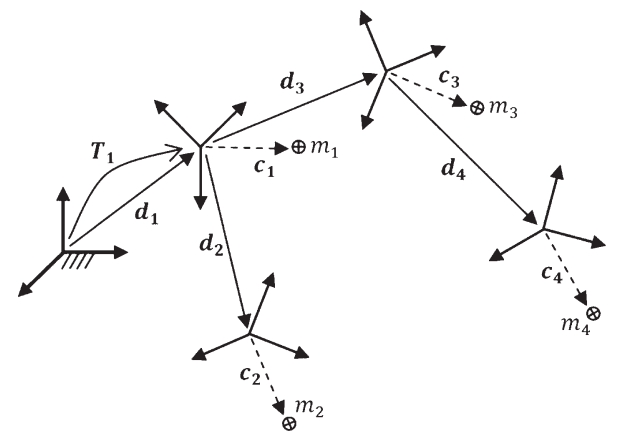

(a) Tree Chain

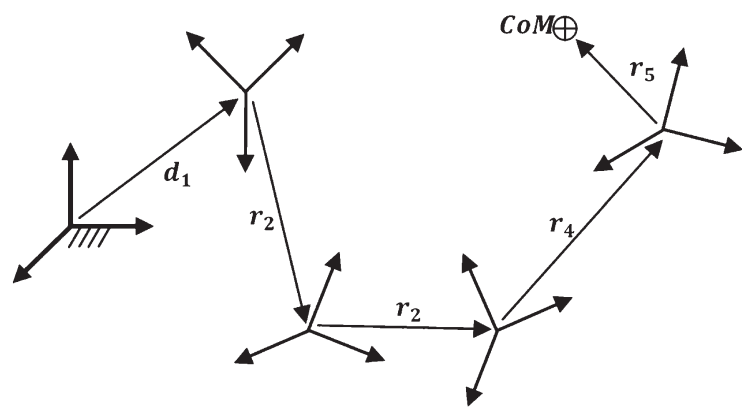

(b) SESC

Fig. 2. (a) represents a four degree of freedom tree chain and (b) represents the statically equivalent serial chain locating the CoM of the original chain (a).

$$
\left\{\begin{array}{c}
C o M \\
1
\end{array}\right\}=\frac{m_{1}}{M} \mathbf{T}_{1}\left\{\begin{array}{c}
c_{1} \\
1
\end{array}\right\}+\frac{\mathbf{m}_{\mathbf{2}}}{\mathbf{M}} \mathbf{T}_{\mathbf{1}} \mathbf{T}_{\mathbf{2}}\left\{\begin{array}{c}
c_{2} \\
1
\end{array}\right\}+\frac{\mathbf{m}_{3}}{\mathbf{M}} \mathbf{T}_{\mathbf{1}} \mathbf{T}_{\mathbf{3}}\left\{\begin{array}{c}
c_{3} \\
1
\end{array}\right\}+\frac{\mathbf{m}_{4}}{\mathbf{M}} \mathbf{T}_{\mathbf{1}} \mathbf{T}_{\mathbf{3}} \mathbf{T}_{\mathbf{4}}\left\{\begin{array}{c}
c_{4} \\
1
\end{array}\right\}
$$

Expanding,

$$
\begin{aligned}
C o M= & d_{1}+\mathbf{A}_{1} r_{2}+\mathbf{A}_{1} \mathbf{A}_{2} r_{3}+\mathbf{A}_{1} \mathbf{A}_{3} r_{4} \\
& +\mathbf{A}_{1} \mathbf{A}_{3} \mathbf{A}_{4} r_{5}
\end{aligned}
$$

$$
\text { where }
$$

$$
\begin{aligned}
& \boldsymbol{r}_{\mathbf{2}}=\left(m_{1} \boldsymbol{c}_{\mathbf{1}}+m_{2} \boldsymbol{d}_{\mathbf{2}}+\left(m_{3}+m_{4}\right) \boldsymbol{d}_{\mathbf{3}}\right) / M \\
& \boldsymbol{r}_{\mathbf{3}}=\left(m_{2} \boldsymbol{c}_{\mathbf{2}}\right) / M \\
& \boldsymbol{r}_{\mathbf{4}}=\left(m_{3} \boldsymbol{c}_{\mathbf{3}}+m_{4} \boldsymbol{d}_{\mathbf{4}}\right) / M \\
& \boldsymbol{r}_{\mathbf{5}}=\left(m_{4} \boldsymbol{c}_{\mathbf{4}}\right) / M
\end{aligned}
$$


Observe that with a complete knowledge of the kinematic and static parameters of the system, the $\boldsymbol{r}_{\boldsymbol{i}}$ vectors in Equation (4) are known. Moreover, for a system connected by only revolute and spherical joints, the $\boldsymbol{d}_{\boldsymbol{i}}$ are constant and, thus, the $\boldsymbol{r}_{i}$ are too. Letting $\mathbf{A}_{1}^{*}=\mathbf{A}_{1}$, $\mathbf{A}_{2}^{*}=\mathbf{A}_{1} \mathbf{A}_{2}, \mathbf{A}_{3}^{*}=\mathbf{A}_{1} \mathbf{A}_{3}$, and $\mathbf{A}_{4}^{*}=\mathbf{A}_{1} \mathbf{A}_{3} \mathbf{A}_{4}$, the similarity between the expression in Equation (5) and the forward kinematics of the serial chain depicted in Fig. 2(b) is noted,

$$
C o M=d_{1}+A_{1}^{*} r_{2}+A_{2}^{*} r_{3}+A_{3}^{*} r_{4}+A_{4}^{*} r_{5}
$$

The result is that the CoM location of the original branched chain is modelled by the end-effector location of an appropriately sized spatial serial-chain, maintaining the same DOF as the original branched chain.

For the purposes of estimating the centre of mass location, Equation (3) is manipulated in yet another way,

$$
\boldsymbol{C o M}=\left[\begin{array}{lllll}
I & \mathrm{~A}_{1}^{*} & \mathrm{~A}_{2}^{*} & \mathbf{A}_{3}^{*} & \mathbf{A}_{4}^{*}
\end{array}\right]\left\{\begin{array}{l}
d_{1} \\
r_{2} \\
r_{3} \\
r_{4} \\
r_{5}
\end{array}\right\}
$$

where $\mathbf{I}$ is the $3-b y-3$ identity matrix. This concept can be applied to any multi-link chain. If the multilink chain contains only revolute and spherical joints, the vector composed from the concatenation of the $\boldsymbol{r}_{\boldsymbol{i}}$ vectors is a constant. Thus, Equation (6) can be written in the form:

$$
\operatorname{CoM}=\left[\begin{array}{llll}
\mathrm{I} & \mathrm{A}_{1}^{*} & \cdots & \mathrm{A}_{\mathrm{n}}^{*}
\end{array}\right]\left\{\begin{array}{c}
d_{1} \\
r_{2} \\
\vdots \\
r_{n+1}
\end{array}\right\}=\mathrm{B} R
$$

where $n$ is the degrees of freedom of the original chain, the vector $\boldsymbol{R}$ is constant, and the matrix $\mathbf{B}$ is 3 -by-3(n+1) for the spatial case.

\subsection{Statically equivalent serial chain estimation}

Assuming that the mechanical parameters of the system under study are partially or totally unknown (as in the case of a human subject), the SESC parameters $\left(\boldsymbol{r}_{i}\right.$ vectors) are also unknown. However, they can be estimated from multiple recordings of the system in different configurations. Consequently, the CoM can be estimated.

\subsubsection{Constructing the SESC from CoM data}

For simplicity, assume that the fixed frame of the system is aligned with the first joint, or $\boldsymbol{d}_{\mathbf{1}}=\mathbf{0}$, and Equation (7) simplifies to:

$$
\boldsymbol{C o M}=\left[\begin{array}{lll}
\mathbf{A}_{1}^{*} & \cdots & \mathbf{A}_{\mathrm{n}}^{*}
\end{array}\right]\left\{\begin{array}{c}
r_{2} \\
\vdots \\
r_{n+1}
\end{array}\right\}=\mathrm{B} \boldsymbol{R}
$$

For this case, the matrix $\mathbf{B}$ is $3-b y-3 n$. For a given configuration of the body, configuration $i$, the position of the CoM can be expressed as $\boldsymbol{C o} \boldsymbol{M}_{\boldsymbol{i}}=\mathbf{B}_{\mathbf{i}} \boldsymbol{R}$. Hence, for $m$ configurations of the body, with $m \geq n$

$$
\left\{\begin{array}{c}
C o M_{1} \\
\vdots \\
\operatorname{CoM}_{m}
\end{array}\right\}=\left[\begin{array}{c}
\mathrm{B}_{1} \\
\vdots \\
\mathrm{B}_{\mathrm{m}}
\end{array}\right] \mathbf{R}=\mathrm{DR}
$$

The matrix $\mathbf{D}$ is $3 m-b y-3 n$. Even though $\mathbf{D}$ is not full rank, the vector containing the center of mass locations is in its range space, and there exists a solution for $\boldsymbol{R}$ or, to be more precise, many solutions for $\boldsymbol{R}$. One of these solutions may be determined using the pseudo-inverse, and determines the parameters of a SESC.

$$
R=\mathrm{D}^{+}\left\{\begin{array}{c}
\operatorname{CoM}_{1} \\
\vdots \\
\operatorname{CoM}_{m}
\end{array}\right\}
$$

Given $\boldsymbol{R}$, Equation (8) could then be used to determine the CoM for any other configuration of the body. The non uniqueness of the SESC is due to the fact that several choices of kinematic parameters yield serial chain manipulators capable of producing the same endeffectors location.

\subsubsection{Onstructing the SESC from partial CoM data}

The problem with the procedure previously described is that the general CoM is not readily known. However, it is possible to determine the vector $\boldsymbol{R}$ if some information on the position of the CoM is available; for example if the coordinates of its projection on the ground are known. Consider the known components to be in the $x$ and $y$ directions, i.e. horizontal directions (where there is no indication of the $z$ components of the CoM, i.e. vertical component). Ignoring the unknown component, Equation (8) becomes

$$
\left\{\begin{array}{l}
\operatorname{CoM}_{x} \\
\operatorname{CoM}_{y}
\end{array}\right\}=\left[\begin{array}{ccc}
\mathbf{A}_{1, \mathbf{x}}^{*} & \cdots & \mathbf{A}_{\mathbf{4}, \mathbf{x}}^{*} \\
\mathbf{A}_{\mathbf{1}, \mathbf{y}}^{*} & \cdots & \mathbf{A}_{\mathbf{4}, \mathbf{y}}^{*}
\end{array}\right]\left\{\begin{array}{c}
\boldsymbol{r}_{\mathbf{2}} \\
\vdots \\
\boldsymbol{r}_{\mathbf{5}}
\end{array}\right\}=\left[\begin{array}{c}
\mathbf{B}_{\mathbf{x}} \\
\mathbf{B}_{\mathbf{y}}
\end{array}\right] \boldsymbol{R}
$$


where the matrices $\mathbf{B}_{\mathbf{x}}$ and $\mathbf{B}_{\mathbf{y}}$ are 1-by-3n. For $3 m$ known CoM components corresponding to $3 m$ different postures, Equation (12) is obtained.

$$
\left\{\begin{array}{c}
\operatorname{CoM}_{1, x} \\
\operatorname{CoM}_{1, y} \\
\vdots \\
\operatorname{CoM}_{3 m, x} \\
\operatorname{CoM}_{3 m, y}
\end{array}\right\}=\left[\begin{array}{c}
\mathbf{B}_{\mathbf{1}, \mathbf{x}}^{*} \\
\vdots \\
\mathbf{B}_{\mathbf{3} \mathbf{m}, \mathbf{y}}^{*}
\end{array}\right] \boldsymbol{R}=\mathbf{D} \boldsymbol{R}
$$

Finally,

$$
\boldsymbol{R}=\mathbf{D}^{+}\left\{\begin{array}{c}
\operatorname{CoM}_{1, x} \\
\operatorname{CoM}_{1, y} \\
\vdots \\
\operatorname{CoM}_{3 n, x} \\
\operatorname{CoM}_{3 n, y}
\end{array}\right\}
$$

Again, given $\boldsymbol{R}$, Equation (8) determines the CoM for any other configuration of the body. The collection of $3 n$ pieces of data, in theory, is enough for the procedure to work. Due to the vagaries of the actual data collection in practice, many more such readings are needed $(m>n)$. Additionally, with planar systems, the multiplier of 3 in the above equations is replaced by 2 . That is, in Equation (11) and (12), $\mathbf{B}_{\mathbf{x}}$ is $1-b y-2 n$, and $\mathbf{D}$ is $2 m-b y-2 n$. Intriguingly, this matrix in the planar case is full rank. Again, due to vagaries in the collection of data, many more points are used and the pseudo-inverse remains a necessity.

\subsection{Data collection}

Two sources of information, over different static postures, are required to produce an estimation of the SESC of the subject. The first one is the collection of the joint values of the subject and the second one is the collection of the horizontal components of the subject's centre of mass. As this work is devoted to care-needed and more especially elderly people, a priority was to use common devices, easy to use but, more importantly, not invasive for the subject. To this aim, joint values were collected through motion capture equipment while centre of mass components were collected with force plates.

\subsubsection{Joint values}

Although several types of equipment are available to compute the joint values of a subject, a motion capture system seems one of the best suited to record joint values. In fact, human joints are complex. They are not only revolute but often spherical joints, and in some cases a translation is also associated with their movement. Consequently it is difficult to find equipment able to render accurately the complex rotation of a joint. Thus, we opt for a system capable of analysing the environment of the joint, rather than measuring directly the rotation value. A motion capture system does not measure directly the value of the joint rotation (as would be the case if an electrogoniometer were used) but tracks the position of reflective markers in the space. Knowing the instantaneous position of these markers, attached to the skin of the subject under study, the value of the joint angles can be computed. Figure 3(a) shows a typical subject equipped with markers for a motion capture session. For example, to measure the elbow angle, the two segments used will be the upper arm and the forearm. The motion capture system used for the experiment conducted in this paper is depicted in Fig. 3(b).

\subsubsection{CoM horizontal components}

In subsection 2.2.2, it has been shown that the centre of mass of a subject can be estimated even if its position is partially known (two of the three components are available). This knowledge can be obtained using force plates to measure the forces $\left(F_{x}, F_{y}, F_{z}\right)$ and moments $\left(M_{x}, M_{y}, M_{z}\right)$ exerted by the subject on the ground. Such information allows the computation of the Centre of Pressure ( $\mathrm{CoP})$ position, using Equation (14):

$$
\begin{aligned}
& \operatorname{CoP}_{x}^{F P}=\frac{-\left(M_{y}+F_{x} * Z_{0}\right)}{F_{z}}+X_{0} \\
& \operatorname{CoP}_{y}^{F P}=\frac{\left(M_{x}+F_{y} * Z_{0}\right)}{F_{z}}+Y_{0}
\end{aligned}
$$

where $X_{0}, Y_{0}$ and $Z_{0}$ are the force plate offsets. The CoP given by Equation (14) is expressed in the force plate reference frame $\left(C_{o} P_{x}^{F P}\right)$, and hence a translation is necessary to express it in the reference frame of the subject (i.e. the frame used to express the position of the CoM). The concept is illustrated by Fig. 4, which shows the two reference frames.

In perfectly static conditions, the projection of the CoM corresponds to the CoP, but this is never achieved in practice due to the postural sway typical of human standing. However, when a subject is asked to maintain a chosen posture, the amplitude and frequency of the postural sway are very low, and so is the acceleration of the body. As a result, the forces induced 


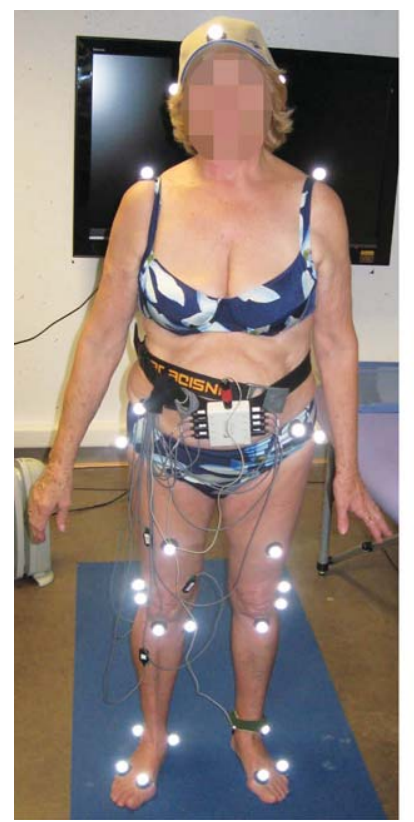

(a) Markers placement

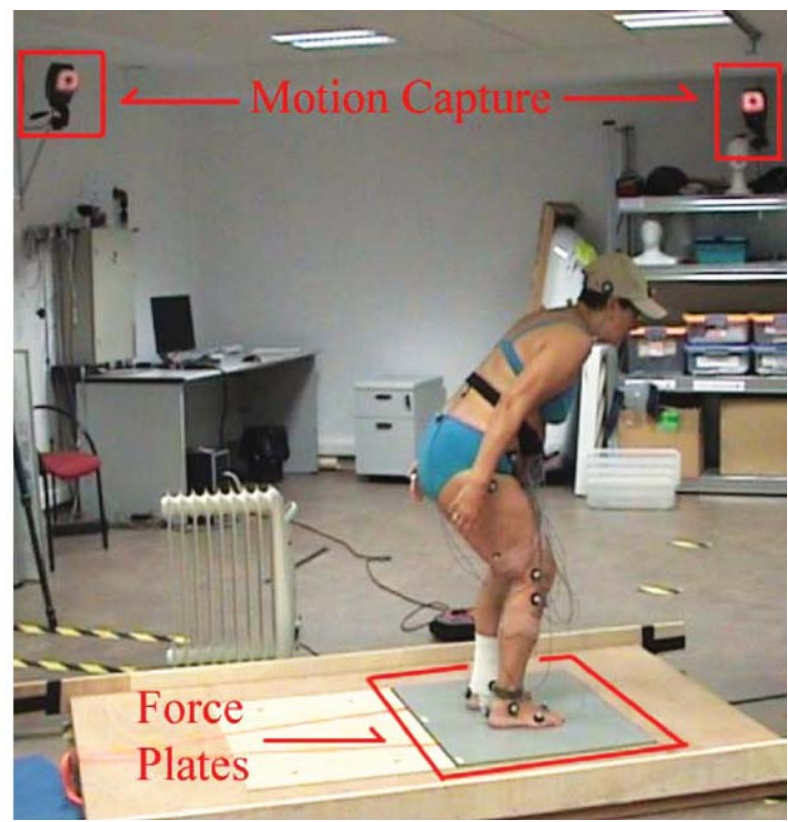

(b) Devices for the CoM Estimation

Fig. 3. Experimental environment: (a) Marker locations on the subject. (b) Motion capture and force plate devices used to estimate the CoM.

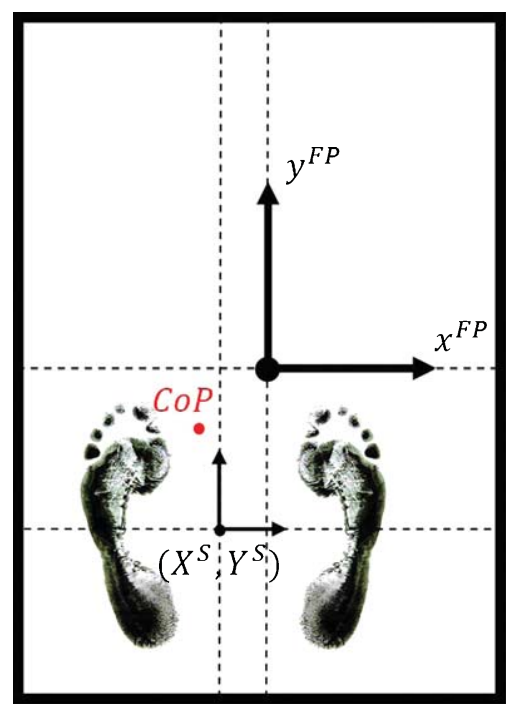

Fig. 4. Difference between force plate and subject reference frames.

by the dynamic of the movement are negligible when compared to those induced by gravity; hence, the CoM projection on the ground is quasi equal to the CoP.
Consequently, the centre of mass horizontal components $\left(\operatorname{CoM}_{x, y}\right)$ are given by Equation (15), where $\left(X_{s}, Y_{s}\right)$ are the coordinates of the subject reference frame in the force plate frame.

$$
\begin{aligned}
& C o M_{x} \approx C o P_{x}^{S}=\operatorname{CoP}_{x}^{F P}-X_{S} \\
& C o M_{y} \approx \operatorname{CoP}_{y}^{S}=\operatorname{CoP}_{y}^{F P}-Y_{S}
\end{aligned}
$$

\subsubsection{Static period tracking}

During the practical experimentations, the participating volunteers were asked to maintain a few chosen poses (described later, in section 3.4.), for five seconds each. This ensured that the CoP position computed from the force plate signals was quasi-equal to the $\mathrm{CoM}$ projection on the ground, under the assumption that the subject was indeed maintaining a static posture. In order to verify this assumption, the CoP position computed from the recordings for each pose was analysed to identify the time window with the minimal standard deviation (a time window of $500 \mathrm{~ms}$ was considered, see Fig. 5). The posture was deemed static if the standard deviation of the CoP position was less than $1 \mathrm{~mm}$. Finally, on this segment of time, the mean of the joint values and the mean of the centre of pres- 


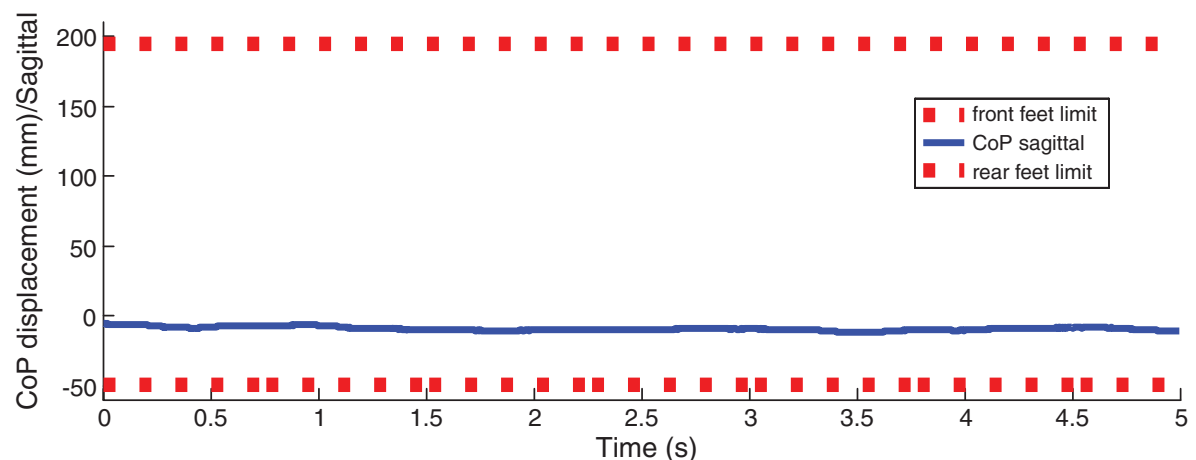

(a)

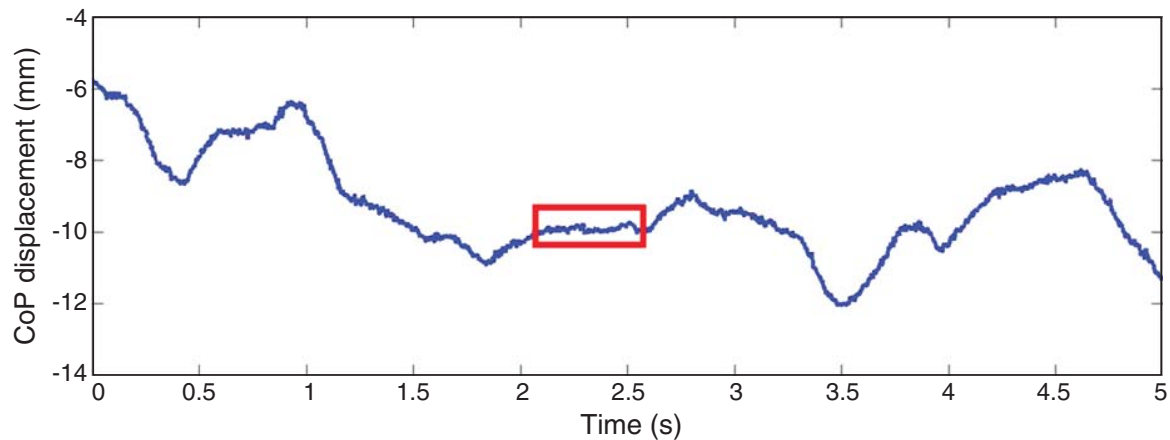

(b)

Fig. 5. Static period tracking. (a) CoP position recorded while the subject is maintaining a static position. (b) Enlarged signal view: the red box indicates the period considered for the SESC identification.

sure were realized. This process was repeated for each static pose.

\section{CoM estimation of elderly people}

\subsection{Study objectives}

The present study considered the estimation of the centre of mass on the sagittal plane. The human body is appropriately modelled on this plane with an open kinematic chain with three rotational links corresponding to the ankle, knee and hip. After applying the estimation process presented in the previous section, a minimal set of required postures, allowing an accurate estimation of the CoM, is presented. Estimation results are finally compared to the classical $\mathrm{CoM}$ calculation using anthropometric tables.

\subsection{Experimental activities}

Experimental tests were conducted on 2 youngs (under 35 years of age) and 9 elderly (over 65 years of age) volunteers. The characteristics of each subject are reported in Table 1. After being briefed on the test procedures and the goals of the study, the subjects signed a consent form to confirm that they had freely agreed to participate.

Table 1

Data collected for a subject on six static poses

\begin{tabular}{lccccc}
\hline & $\begin{array}{c}\text { Age } \\
(\text { years })\end{array}$ & $\begin{array}{c}\text { Gender } \\
\text { Gender }\end{array}$ & $\begin{array}{c}\text { Weight } \\
(\mathrm{Kg})\end{array}$ & $\begin{array}{c}\text { Height } \\
(\mathrm{m})\end{array}$ & $\begin{array}{c}\text { BMI } \\
\left(\mathrm{Kg} / \mathrm{m}^{2}\right)\end{array}$ \\
\hline Subject 1 & 71 & $\mathrm{~F}$ & 68.66 & 1.59 & 27.16 \\
Subject 2 & 70 & $\mathrm{~F}$ & 58.87 & 1.50 & 26.16 \\
Subject 3 & 75 & $\mathrm{M}$ & 69.91 & 1.75 & 22.83 \\
Subject 4 & 84 & $\mathrm{~F}$ & 74.34 & 1.54 & 31.35 \\
Subject 5 & 68 & $\mathrm{M}$ & 71.41 & 1.54 & 30.11 \\
Subject 6 & 34 & F & 55.53 & 1.49 & 25.01 \\
Subject 7 & 75 & M & 80.76 & 1.61 & 31.16 \\
Subject 8 & 78 & F & 62.34 & 1.57 & 25.29 \\
Subject 9 & 66 & F & 69.41 & 1.54 & 29.27 \\
Subject 10 & 75 & M & 56.77 & 1.54 & 23.94 \\
Subject 11 & 23 & M & 80.00 & 1.84 & 23.62 \\
\hline
\end{tabular}


Each volunteer was asked to maintain each of the 6 static poses represented in Fig. 7 for five seconds. A motion capture system was used to record the movement of the body, and two force plates (one under each foot) were used to measure the moments and the forces exerted on the ground.

The motion capture system (Hawk Digital, Motion Analysis Inc.) recorded the position, in 3D space, of 30 passive reflective markers attached to the skin of the subject with double sided, medical grade tape. The high number of markers was required by another investigation on the sit-to-stand movement. However, only a subset of markers was used in this study, where a simplified planar model was considered. The markers considered were those placed on the heels, the malleoli, the first metatarsal heads, the tibial plates, the great throchanters and the acromions. Their position was acquired at a frequency of $200 \mathrm{~Hz}$, and low-pass filtered at $5 \mathrm{~Hz}$ via software (Evart v 5.04, Motion Analysis Inc.). The analog outputs of the force platforms

\subsection{Three degrees of freedom model}

Generally, elderly people cannot perform difficult static poses (on one foot for example) and often their motions amplitude is limited. Consequently they have a poor range of available static poses. To this aim a simple model should be used. As one of the long term goals of the experiment conducted on these elderly subjects is to study their sit-to-stand motions, an estimation of the variation of the centre of mass in the sagittal plane seemed the most appropriate. With these constraints, three joints have been selected to describe the subject motion: ankle, knee and hip. Figure 6 shows the recorded joint values and the associated kinematic model.

The first step in the estimation process described in section 2 consist in expressing the CoM of this three degrees of freedom model as if the mechanical parameters were all perfectly known. The CoM position given by the SESC model is:

$$
\left\{\begin{array}{l}
\operatorname{CoM}_{x} \\
\operatorname{CoM}_{y}
\end{array}\right\}=\left[\begin{array}{llll}
0 & \sin \left(\theta_{1}\right) & \sin \left(\theta_{1}+\theta_{2}\right) & \sin \left(\theta_{1}+\theta_{2}+\theta_{3}\right) \\
1 & \cos \left(\theta_{1}\right) & \cos \left(\theta_{1}+\theta_{2}\right) & \cos \left(\theta_{1}+\theta_{2}+\theta_{3}\right)
\end{array}\right]\left\{\begin{array}{l}
r_{1} \\
r_{2} \\
r_{3} \\
r_{4}
\end{array}\right\}
$$

(AMTI BP400600-1000) were amplified (using two AMTI MiniAmp MSA-6 amplifiers) and converted using an A/D converter (National Instruments USB6218), which also ensured synchronization with the motion capture system. These signals (3 forces and 3 moments for each force plate) were sampled at $2 \mathrm{kHz}$, and low-pass filtered at $40 \mathrm{~Hz}$ with a $2^{\text {nd }}$ order Butterworth filter implemented in Matlab (The Mathworks). Data were recorded not only while the subject maintained the static poses, but also during the transitions between each pose and the following one. This effectively produced two sets of data: one was used for the identification of the SESC (data recorded during the static poses), and the other was used for the comparison of the technique with a "traditional" where

$$
\begin{aligned}
& r_{1}=d_{0}\left(m_{1}+m_{2}+m_{3}\right) / M+m_{0} / M \approx d_{0} \\
& r_{2}=\left(m_{1} c_{1}+d_{1}\left(m_{2}+m_{3}\right)\right) / M \\
& r_{3}=\left(m_{2} c_{2}+d_{2} d_{4} m_{3}\right) / M \\
& r_{4}=\left(m_{3} c_{3}\right) / M
\end{aligned}
$$

Note here that, as reported in Table 5, the mass of the feet counts only for $2.9 \%$ of the total mass of the body, and therefore can be considered negligible. As a result, $r_{1}$ can be considered equal to $d_{0}$. As $d_{0}$, the height of the ankles, is defined by the markers on the malleoli, the complexity of the system can be reduced and, consquently, the number of required postures for the identification can also be reduced. Equation (16) becomes:

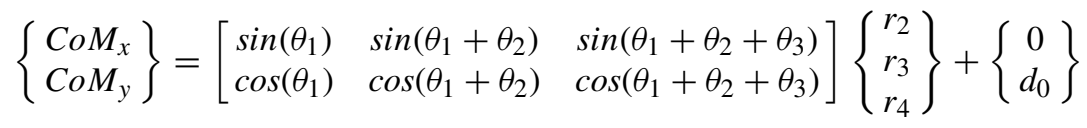

For the purpose of estimation, Equation (18) is manipulated according to section 2.2.2

method for CoM tracking (data recorded during transitions).

$$
\operatorname{CoM}_{x}=\left[\begin{array}{lll}
\sin \left(\theta_{1}\right) & \sin \left(\theta_{1}+\theta_{2}\right) & \sin \left(\theta_{1}+\theta_{2}+\theta_{3}\right)
\end{array}\right]\left\{\begin{array}{l}
r_{2} \\
r_{3} \\
r_{4}
\end{array}\right\}
$$



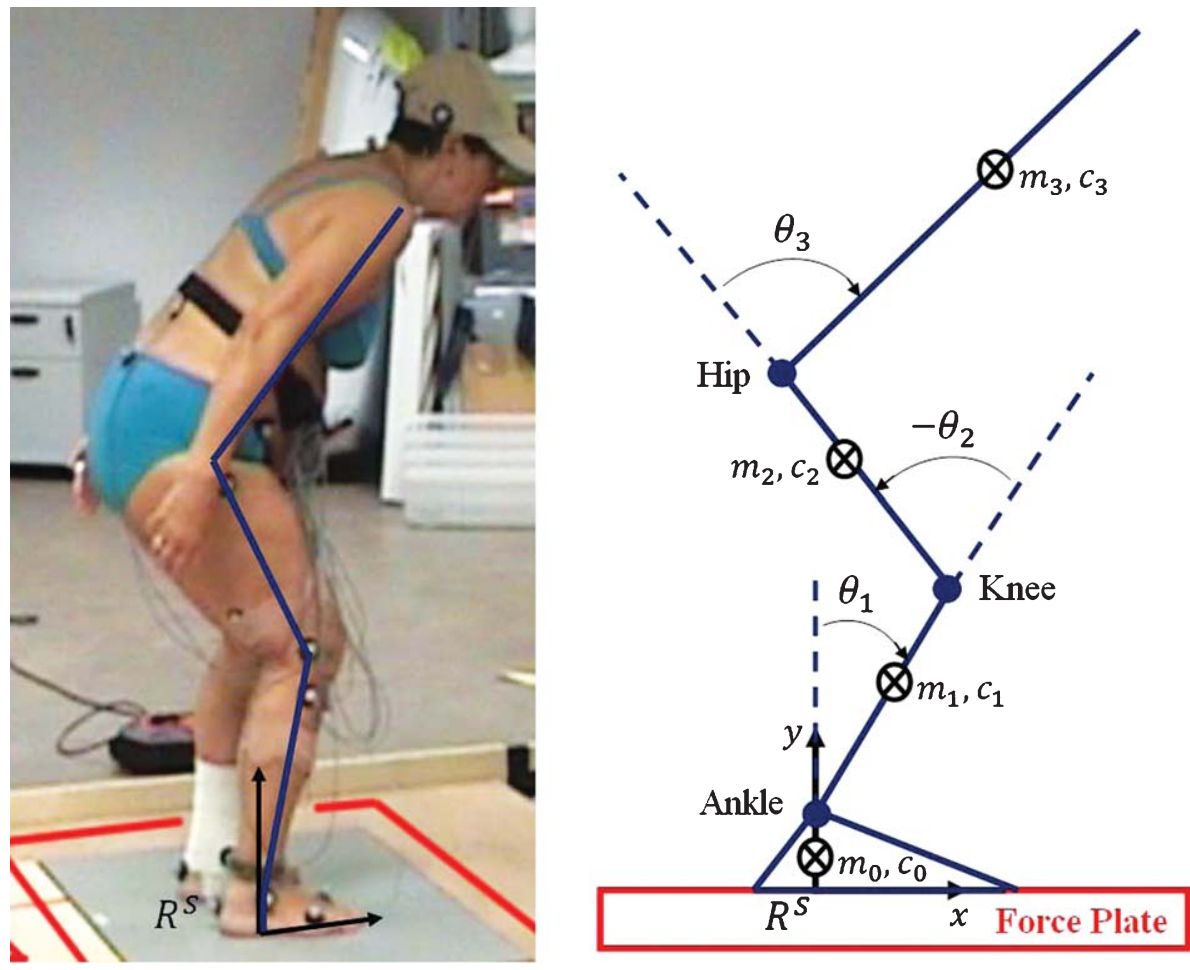

Fig. 6. Kinematic model of the subject used to produce the estimation of its CoM.

With the data collected on the six postures,

$$
\left\{\begin{array}{c}
\operatorname{CoM}_{1, x} \\
\vdots \\
\operatorname{CoM}_{6, x}
\end{array}\right\}=\left[\begin{array}{ccc}
\sin \left(\theta_{1}^{1}\right) & \sin \left(\theta_{1}^{1}+\theta_{2}^{1}\right) & \sin \left(\theta_{1}^{1}+\theta_{2}^{1}+\theta_{3}^{1}\right) \\
\vdots & & \\
\sin \left(\theta_{1}^{6}\right) & \sin \left(\theta_{1}^{6}+\theta_{2}^{6}\right) & \sin \left(\theta_{1}^{6}+\theta_{2}^{6}+\theta_{3}^{6}\right)
\end{array}\right]\left\{\begin{array}{l}
r_{2} \\
r_{3} \\
r_{4}
\end{array}\right\}=\mathbf{D} \boldsymbol{R}
$$

Finally,

$$
\boldsymbol{R}=\mathbf{D}^{+}\left\{\begin{array}{c}
\operatorname{CoM}_{1, x} \\
\vdots \\
\operatorname{CoM}_{6, x}
\end{array}\right\}
$$

Given $\boldsymbol{R}$, Equation (18) determines the CoM for any other configuration of the subject.

\subsection{Required postures}

Theoretically, with the subject's mechanical parameters unknown, three different static postures (three unknowns remain in Equation (18)) are sufficient to estimate his/her centre of mass location. However due to the vagaries of the data collection more static postures are needed. To ensure an accurate estimation, six different postures were selected, see Fig. 7.
The first is a normal standing posture, with the upper limbs placed alongside the trunk (the same upper body posture is maintained in all the 6 positions). The second posture consists in a backwards extension of the trunk, with the lower limbs perpendicular to the ground. The third is a plantar dorsiflexion, resulting in a forward lean of the whole body, with all the joints (except for the ankle) fixed at the same angle adopted during standing. The fourth is a knee flexion, with the trunk perpendicular to the ground, and the fifth is a forward trunk flexion, maintaining the lower limbs as in the standing position. Finally, the sixth posture is obtained through a concomitant flexion of the knees and forward flexion of the trunk. These postures were selected taking into account the poor range of available static poses of elderly people and using the sensitivity analysis of the estimation process presented in [5]. 

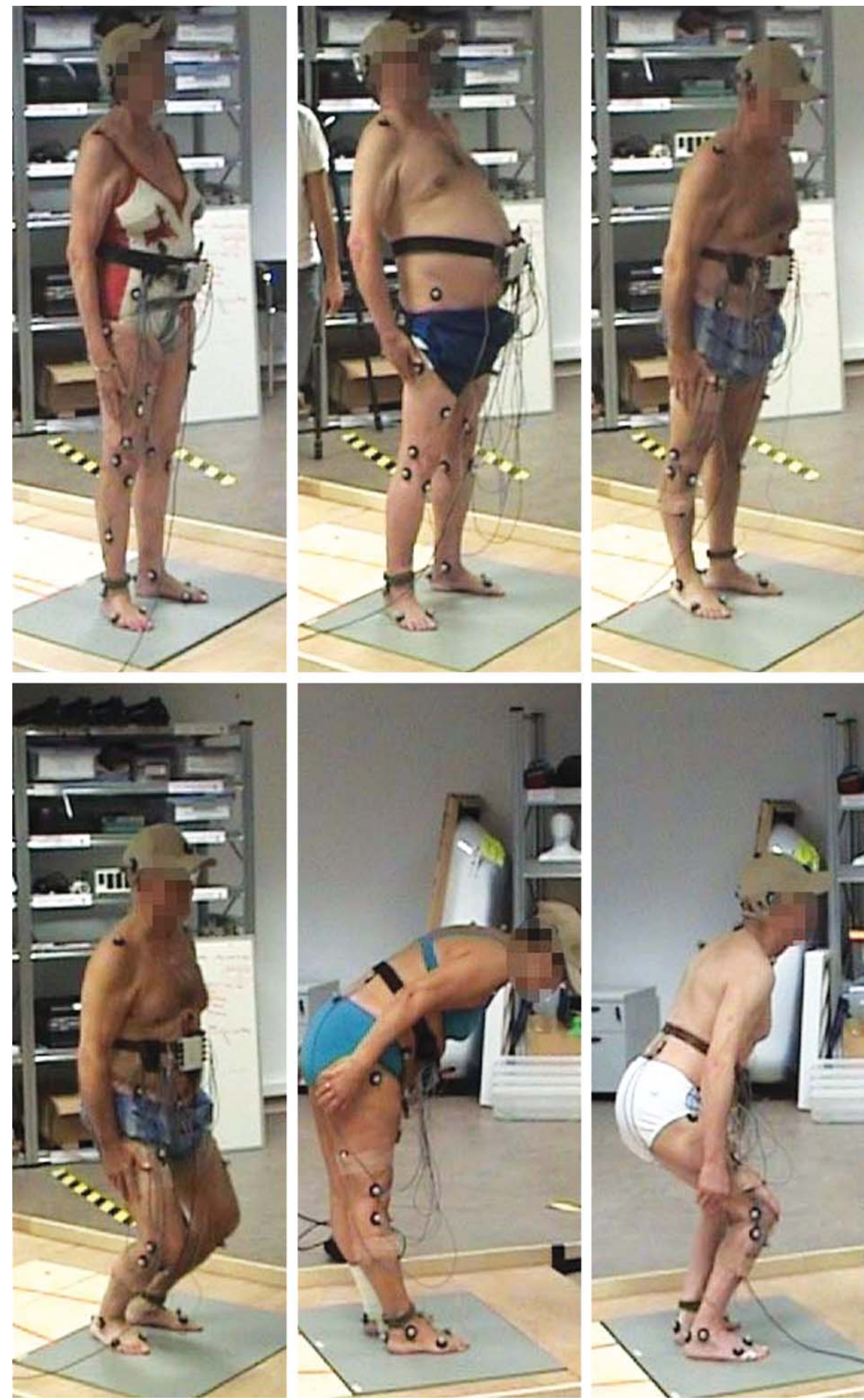

Fig. 7. Six static poses required to identify the SESC locating the subject's CoM 


\subsection{Experimental results}

The SESC of each subject was estimated from the data collected on the six static poses. Only the horizontal component of the centre of mass can be read from force plates. Table 2 gives an example of the data collected for a subject, which were used to estimate its statically equivalent serial chain. Table 3 gives the segment length of the estimated SESC of each subject.

\subsection{Validation of the estimation process}

As described in previous publications $[5,6]$, the CoM estimation technique based on a SESC was validated on a HOAP3 humanoid robot. This was a particularly appropriate platform for the estimation validation, because the mechanical parameters of the robot and thus its CoM location are exactly known (from manufacturer data). Clearly, in the case of an application to human beings as the one presented in this paper, such a direct comparison of estimated and real CoM positions is not possible. However we can compare the estimated CoM horizontal components to the CoP in static cases and to other estimation techniques in non static cases. The vertical component will

Table 2

Data collected for a subject on six static poses

\begin{tabular}{lrrrr}
\hline Pose & Ankle & \multicolumn{1}{c}{ Knee } & Hip & CoP $(\mathrm{mm})$ \\
\hline 1 & 2.1651 & -3.0141 & 13.6797 & 52.1 \\
2 & 2.3168 & 3.9114 & 15.8258 & 110.5 \\
3 & -8.4628 & 5.1964 & 45.1664 & 51.8 \\
4 & 3.5903 & -5.4899 & 4.6462 & 29.2 \\
5 & 40.8426 & -67.7563 & 39.4578 & 104.3 \\
6 & 28.8505 & -57.4122 & 70.5949 & 107.6 \\
\hline
\end{tabular}

Table 3

Segment length of the estimated SESC of each subject

\begin{tabular}{lcccc}
\hline Subject & $d_{0}(\mathrm{~cm})$ & $r_{2}(\mathrm{~cm})$ & $r_{3}(\mathrm{~cm})$ & $r_{4}(\mathrm{~cm})$ \\
\hline 1 & 7.7 & 38.4 & 29.0 & 15.6 \\
2 & 6.9 & 41.5 & 46.4 & 17.7 \\
3 & 7.5 & 43.8 & 34.8 & 20.3 \\
4 & 7.0 & 42.2 & 39.1 & 19.5 \\
5 & 6.1 & 32.6 & 33.3 & 17.2 \\
6 & 6.3 & 35.8 & 31.4 & 18.1 \\
7 & 7.9 & 30.0 & 26.7 & 20.2 \\
8 & 6.3 & 35.5 & 33.8 & 16.1 \\
9 & 7.1 & 31.8 & 28.3 & 14.7 \\
10 & 7.1 & 23.8 & 25.2 & 14.2 \\
11 & 7.5 & 53.4 & 43.4 & 17.6 \\
\hline
\end{tabular}

be compared to results obtained from anthropometrice tables (Winter, Table 5).

\subsubsection{Validation of the estimation of the CoM horizontal components}

In order to test if the CoM estimation performed in this study was acceptable, the projection on the ground of the estimated CoM (with anthropometric tables and with the method presented here) were compared with the CoP obtained from the force platforms on new static poses. Figure 8 illustrates typical estimation results. CoP and CoM estimated with SESC method always coincide while anthropometric tables introduce a large error in the CoM estimation.

The CoM estimation was also validated and compared to other well-known estimation techniques during an oscillating motion with a frequency of $0.4 \mathrm{~Hz}$ in the sagittal plane. As indicated in the introduction, two methods are able to estimate accurately the CoM horizontal component of a subject during a motion. These methods are the Low Pass Filter (LPF) [2] and the Second Integral (SI) [13]. However these methods are restricted to horizontal CoM estimation and constrain the subject to stay on the force plates (Subject is not free to move like with the CoM estimation based on the SESC). Figure 9 shows typical estimation results during the oscillating motion. One more times, CoM estimation based on SESC, LPF and SI, gives similar results while anthropometric tables introduce an estimation error.

To check the validity of the CoM estimation on all the subjects, the distance between the real CoP computed from the platforms and the projection of the CoM estimated from the joint angles was considered as an index of acceptability for the estimation. Such a distance was computed for each of the subjects and for each of the new static positions. Table 4 shows the Root Mean Square (RMS) of the difference between $\mathrm{CoP}$ and CoM (computed over 6 new static postures).

Consequently, with such RMS, the estimation process is able to locate accurately the centre of mass in static cases for all the subjects.

Table 4

RMS of the difference between the projection of the estimated CoM on the ground and the CoP computed from the force platforms, on six new static poses

\begin{tabular}{lccccccccccc}
\hline Subject & 1 & 2 & 3 & 4 & 5 & 6 & 7 & 8 & 9 & 10 & 11 \\
\hline RMS (mm) & 0.79 & 1.2 & 1.6 & 2.9 & 5.9 & 2.3 & 3.3 & 1.8 & 3.0 & 5.2 & 0.6 \\
\hline
\end{tabular}




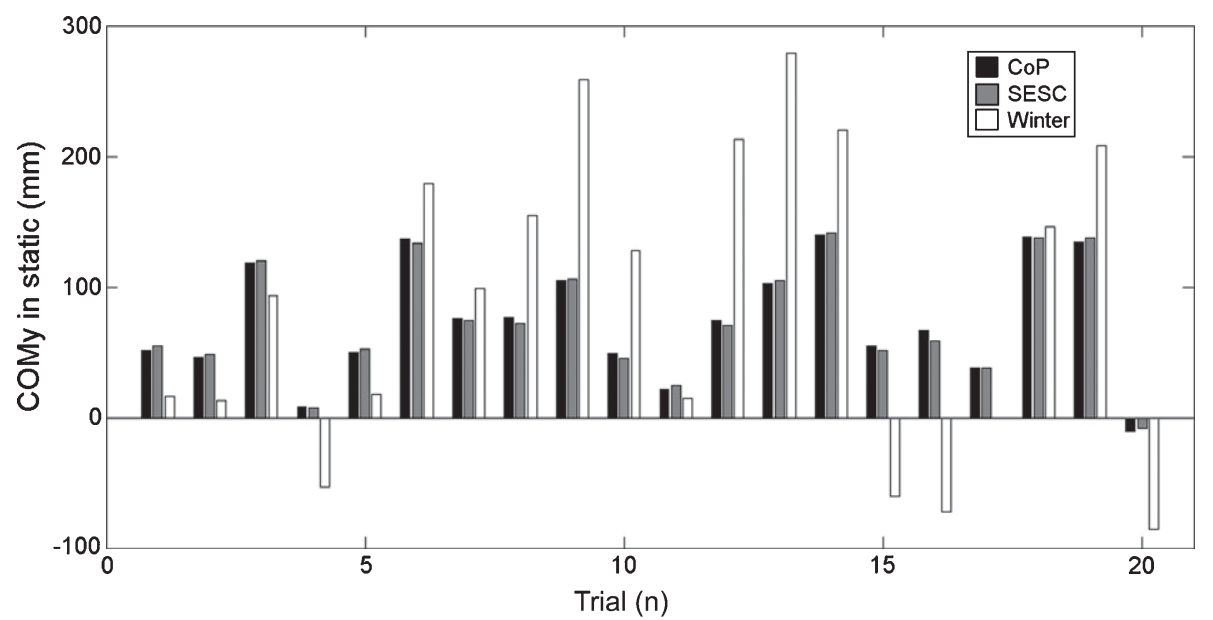

Fig. 8. CoP vs two methods to estimate the CoM on twenty static poses. Only CoM estimation method based on SESC gives accurate results. CoM estimation based on anthropometric tables (Winter, Table 5) introduce large errors.

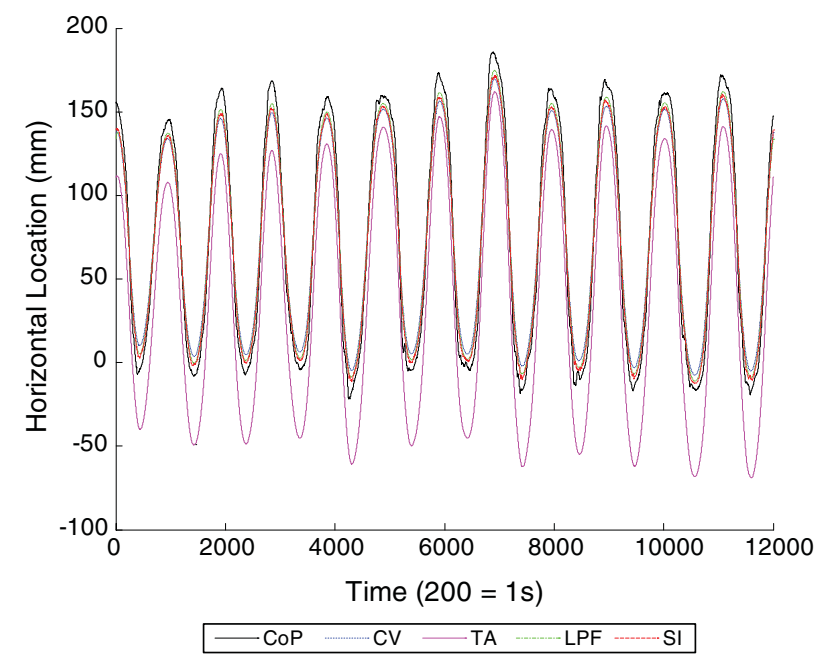

(a) Oscillating motion

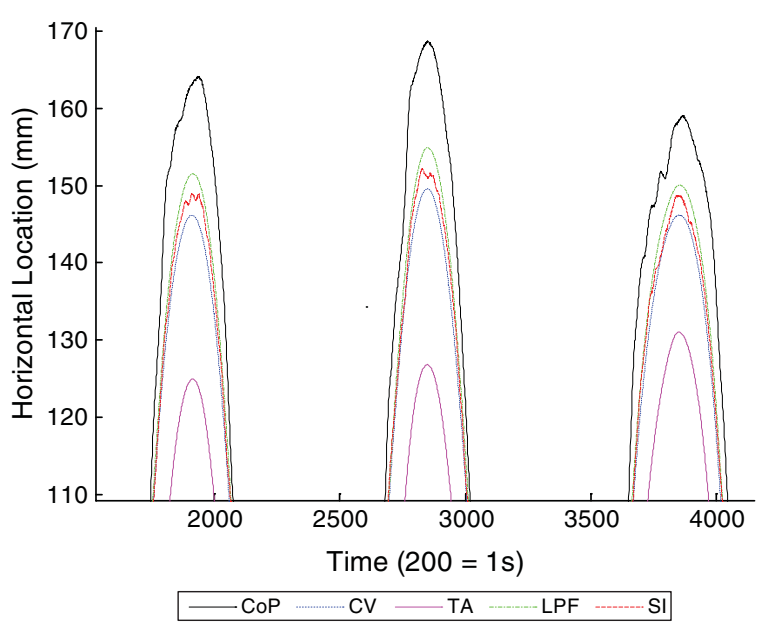

(b) Enlarged view

Fig. 9. CoM estimation during an oscillating motion. LPF = Estimation based on Low Pass Filter method. CV = Estimation based on SESC method. TA = Estimation based on anthropometric tables. SI = Estimation based on Second Integral method.

\subsubsection{CoM estimation using anthropometric tables vs the SESC method}

The only other estimation technique available for providing horizontal and vertical centre of mass locations is the classical formulation of the centre of mass based on the knowledge of the mechanical parameters of the subject. The estimation presented in this paper is now compared to the classical centre of mass calculation, the latter using anthropometric tables to determine
Table 5

Mechanical parameters for the classical CoM calculation

\begin{tabular}{lccc}
\hline Segment & $\begin{array}{c}\text { Segment } \\
\text { Weight }\end{array}$ & $\begin{array}{c}\text { Segment } \\
\text { Length }\end{array}$ & $\begin{array}{c}\text { CoM/Segment } \\
\text { Length }\end{array}$ \\
\hline Foot $^{\mathrm{a}}$ & $0.0145 \mathrm{M}$ & $0.039 \mathrm{H}$ & 0.5 \\
Leg $^{\mathrm{a}}$ & $0.0465 \mathrm{M}$ & $0.246 \mathrm{H}$ & 0.567 \\
Thigh $^{\mathrm{a}}$ & $0.100 \mathrm{M}$ & $0.245 \mathrm{H}$ & 0.567 \\
HAT $^{\mathrm{b}}$ & $0.678 \mathrm{M}$ & $0.470 \mathrm{H}$ & 0.374 \\
\hline
\end{tabular}

${ }^{\mathrm{a}}$ Their weight is counted twice.

${ }^{\mathrm{b}}$ Head, Arms and Trunk. 
a subject's mechanical parameters. The anthropometric tables selected were those from [26] which are largely used in biomechanical studies.

The CoM position is estimated with the two methods for each of the 11 subjects who participated in the study, during a dynamic movement consisting of the continuous transition from one of the static poses required by the identification process to the following one. Figures 10-12 show typical results for elderly and young subjects. For each subject, three plots are reported: the CoM estimation on the horizontal axis with the two methods (Fig. 10(a) and (b)), the CoM estimation on the vertical axis (Fig. (c) and (d)) and the RMS of the difference bewteen the two methods (in this case computed in each instant of time), for the two axes (Figure (e) and (f)). The three plots consider the whole duration of the dynamic movement. The difference between the two estimations was computed over the whole duration of the dynamic movement, in the horizontal and in the vertical direction. Subsequently, the Root Mean Square (RMS) and the standard deviation of each difference were computed. The values for each subject are shown in Figs 11 and 12.

\section{Discussion}

The error in the estimation of the CoP using the SESC method was found to be comparable to that obtained in previous study where the same technique was employed [6]. The RMS of the difference, reported in Table 4, ranges from a value of $0.79 \mathrm{~mm}$ (subject 1) to $5.9 \mathrm{~mm}$ (subject 5). All these values are in an acceptable range; the subject to subject variation may be due to measurement errors. It is important to note that the algorithm for the identification of the SESC uses as inputs the joint angles and the CoP position obtained from platform data. These are used to estimate the CoM position, and its projection on the surface of the force plates is compared to the CoP position used as input. Therefore, the process is insensitive to any error in the measurement taken with the force plates, and the only source of the error reported in Table 4 is the measurement of the joint angles, which were obtained from the 3D markers positions acquired with the motion capture system. The error associated with the marker position depends partly on the motion capture system (this part of error is the same for all the subjects), and partly on factors which are subject-specific: positioning of the markers, soft tissue motion artifacts, physiologi- cal variations in the position of the reference points (malleoli, femoral epycondyle, etc.).

The validity of the estimation given by the SESC could potentially depend on the ability of the subject to maintain the 6 poses required by the identification procedure. In particular, such poses should be static, and characterized by joint angles which are well differentiated among the poses, so that no pose is too similar to another. This could be a potential problem for elderly people, due to a restricted range of motion and increased difficultly in maintaining static equilibrium and prolonged isometric muscle contractions. Interestingly, the results reported in Table 4 do not show a dependency of the performance of the SESC based method from the age of the subject. In fact, the RMS of the error for the young subject (subject 6) was not lower than those found for the elderly subjects. This shows that the method can be used with success even in a study on the elderly population, despite the limitations on the range of static postures which can be safely employed.

The comparison of the CoM estimation conducted with the proposed SESC based method and a classical method based on anthropometric tables reveals an interesting result which is not specific to a study on elderly people. In fact, the use of anthropometric Tables produces an error in the CoM estimation which is higher in the vertical direction than in the horizontal direction (in some cases the difference is as high as an order of magnitude, see Figs 11 and 12). Such a result should be taken into consideration in any study involving CoM estimation using anthropometric tables. Therefore, it may be of interest for a wider scientific audience, and not only for researchers in the field of gerontology.

The results of the comparison also show that the method based on anthropometric Tables introduces an error in the CoM estimation which is not homogeneous across the different subjects.

In the light of the results of previous studies $[5,6]$, the CoM estimation obtained with the SESC method can be considered as a reference in the comparison with an estimation based on anthropometric tables. Therefore, estimation errors between the two methods can be interpreted as a measure of the discrepancy between the body mass distribution predicted by the tables and the real mass distribution for the specific subject. The data presented in this paper show that, at least for the sample of elderly subjects considered in the study (see Fig. 12), there was a high variability of the estima- 


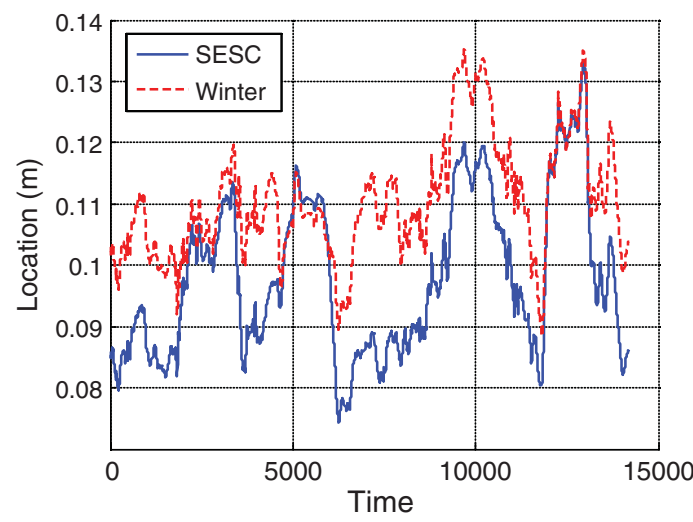

(a) Horizontal - Elderly

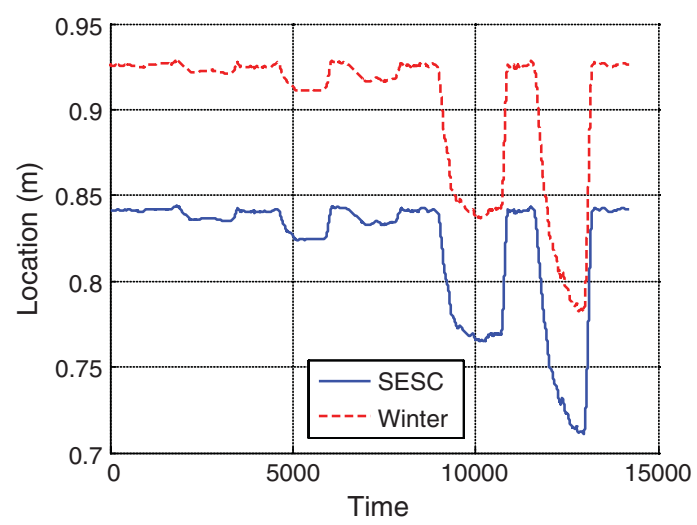

(c) Vertical - Elderly

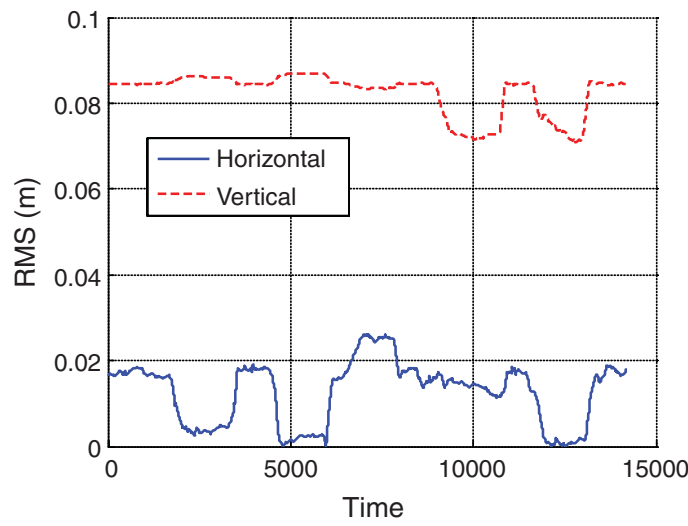

(e) RMS - Elderly

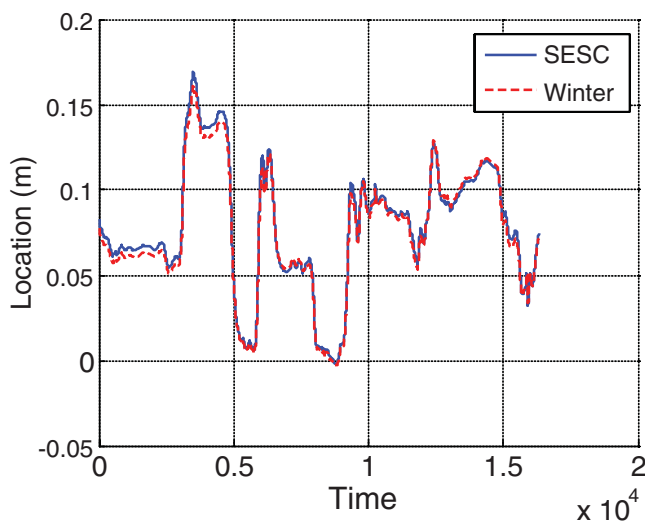

(b) Horinzontal - Young

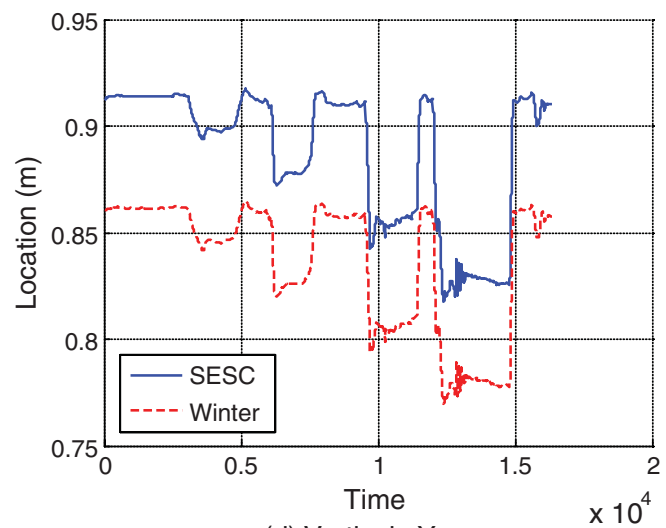

(d) Vertical - Young

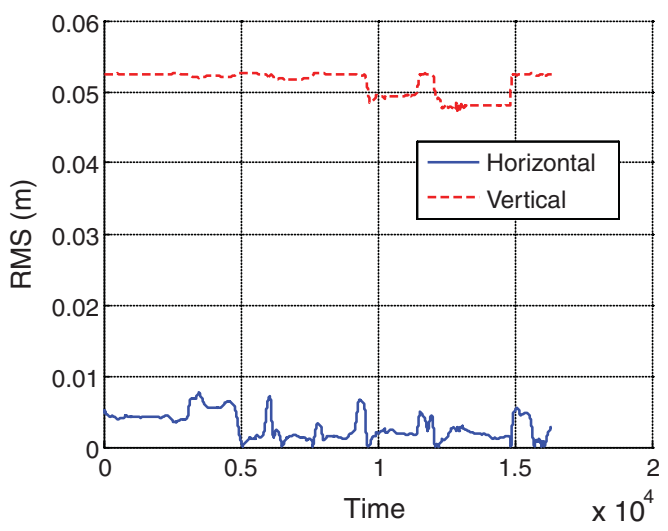

(f) RMS - Young

Fig. 10. CoM Estimation of an elderly and an young subject and, Experiment Results: Comparison of the estimated CoM with the CoM computed classicaly from anthropometric tables; (a) and (b) show the horizontal estimation of the CoM; (c) and (d) show the vertical estimation of the $\mathrm{CoM}$; (e) and (f) show the RMS of the difference between the two estimation methods.

tion error. This is in line with the observations of other authors [4], suggesting that the changes due to ageing produce modifications in the body mass distribution which are not homogeneous across individuals. This result in a higher variability on body proportions of the elderly, making the use of anthropometric tables par- 


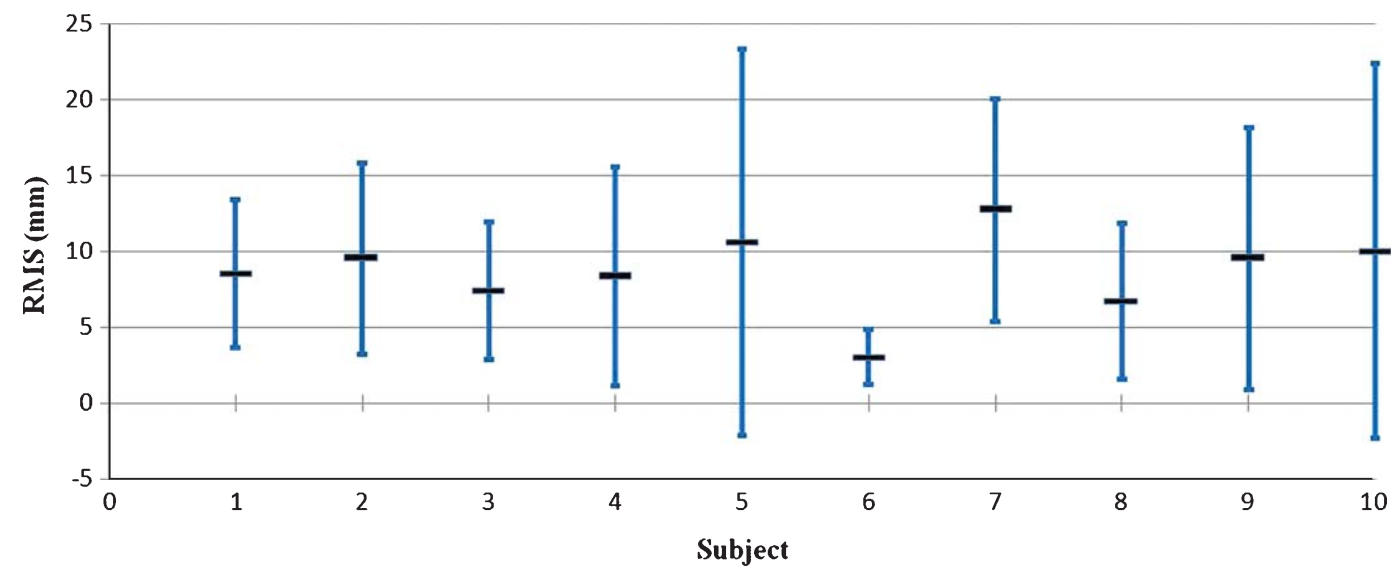

Fig. 11. RMS and standard deviation of the error between CoM estimation based on a SESC and CoM estimation based on anthropometric tables - Horizontal.

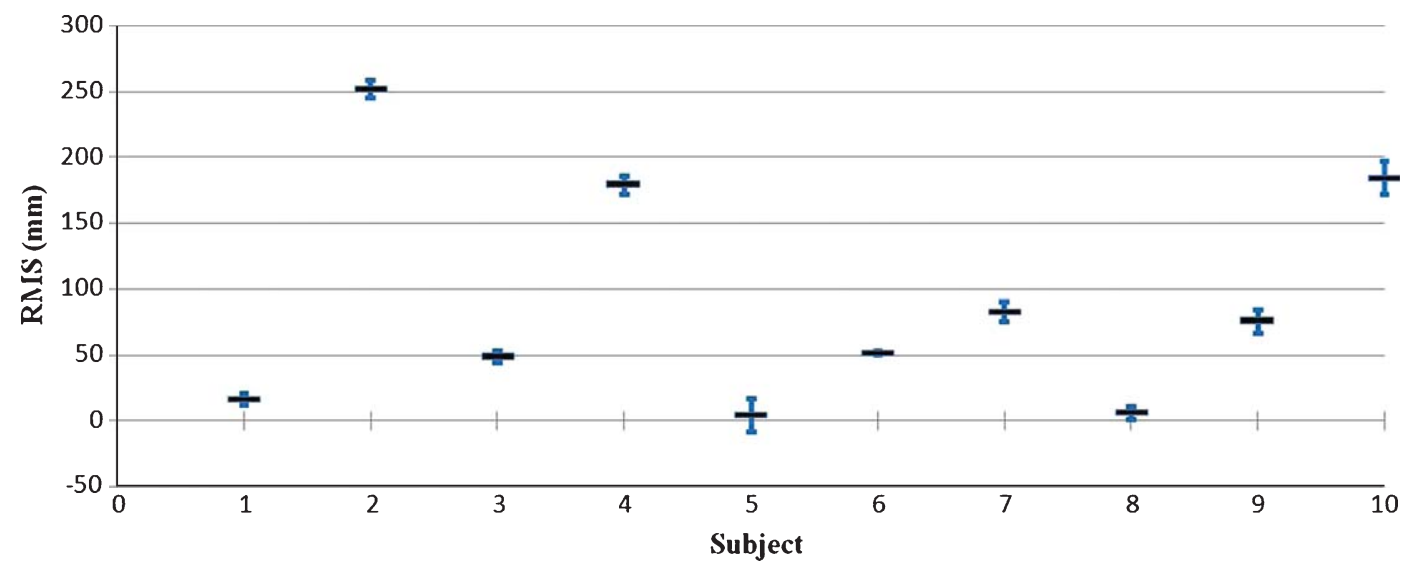

Fig. 12. RMS and standard deviation of the error between CoM estimation based on a SESC and CoM estimation based on anthropometric tables - Vertical.

ticularly problematic in biomechanical studies on the elderly.

Not only are the current anthropometric tables inappropriate, but they would be difficult to modify to accurately describe such an heterogeneous population. Therefore, the estimation of the CoM position based on a SESC is particularly advantageous in biomechanical studies in the field of gerontology. Finally, it is interesting to note that the variation of the error between subjects is not linked to the accuracy of the SESC method evaluated alone (Table 4): the subjects for whom the SESC gives better results (low value in Table 4) are not those for whom the CoM estimation error between the two methods is higher (higher value in Fig. 12. This means that the values reported in Fig. 11 and 12) are not introduced by the SESC based method.
The typical plots of CoM estimation with the two different methods reported in Fig. 10 support the findings described above. In particular, the higher error introduced in the CoM estimation in the vertical direction is evident from the plots of the RMS of the errors between the two methods in the two directions (Fig. 10(e) and (f)).

\section{Conclusions}

In this paper, a novel technique for the estimation of the CoM in human subjects has been applied to a study on an elderly population. The technique, based on the identification of a statically equivalent serial chain, or SESC, allows the estimation of the centre of mass of a subject, from joint angle values, without resorting 
to anthropometric tables. The method proposed relies on an identification process, to be conducted at the beginning of a test, which requires the measurement of joint angles and forces exerted on the ground during 6 static poses.

The SESC based method offer several advantages over other methodologies for the estimation of the CoM in human subjects. Considering the estimation of the CoM projection on the ground, the SESC method gives results which are comparable to those obtained using other validated methods, such as LPF and SI. However, these have some important limitations, which are overcome by the SESC approach. Firstly, the SESC method is suitable for a use in real time, and for movements which are not necessarily periodic. Secondly, and more importantly for the scope of this study, it can be used in test situations requiring the subject to change his or her location in the space. Since LPF and SI are based on force plate measurements, they cannot be used for an estimation of the CoM projection during a displacement. An alternative solution in such a case would be to rely on an estimation based on anthropometric tables and on the measurent of the joint angles, but this method is characterized by a higher estimation error (as reported in previous publications by our group, and summarized in this paper).

The use of anthropometric tables is also the only known alternative to the SESC method when an estimation of the CoM position in the space (and not only its projection on the ground) is required. However, the SESC allows for a real subject-specific estimation, hence not relying on the hypothesis that the subject under study has the same body proportions and mass distribution as those indicated in the tables. The effectiveness in the SESC method for the 3D estimation of the CoM position can be shown on a humanoid robot, for which the CoM position can be precisely computed; in the case of human beings, such validation is not possible, since the CoM position is unknown. However, the comparison between the method based on the SESC and the method based on anthropometric tables for the 2 dimensional case (i.e. projection on the ground) has shown the higher precision of the former. Since the error in the two dimensional estimation is inevitably propagated to the spatial case, the SESC will allow for a more precise 3D estimation as well.

This study presents the first application to the elderly population, and provides two important, practical results. Firstly, it was found that the technique can be successfully employed on the population con- sidered. This is not a trivial conclusion, since only a practical experimentation could show that the SESC identification process was suitable for elderly subjects. In fact, it was initially hypothesized that elderly people would find it difficult to maintain the static postures required by the identification, due to the restricted range of motion and the decreased ability to maintain equilibrium. Secondly, the study showed that the traditional method for the estimation of the CoM based on anthropometric tables introduces an error which characterized by a high subject-to-subject variability. This suggest that anthropometric tables cannot provide an accurate estimation of the parameters of a biomechanical model for elderly people. Hence, not only ageing induces a modification of the distribution of the body masses with respect to younger age, but also a higher variability of such a distribution between subjects.

Therefore, studies on the control of the CoM in later life will particularly benefit from a method characterized by a subject-specific model identification which does not take as inputs only the height and weight (which is the case of anthropometric tables).

The work presented in this paper can be considered as a basis for further investigation on the study of the CoM in elderly people. The use of a SESC method allows a more accurate estimation of the CoM position, and only requires the measurement of the joint angles of interest. Such a measurement can be obtained with a motion capture system, as in the case presented here. However, it can also be obtained with a system based on electrogoniometers or gyroscopes. These devices are minimally invasive for the subject, and relatively easy to use for the researcher. This means that the SESC method allows an accurate tracking of the CoM position to be obtained during daily activities, and not necessarily in a closed environment. Therefore, the proposed technique opens new possibilities for further investigations on the CoM control in elderly people, in the typical environments encountered in everyday life. Further knowledge in the field could be of interest, for example, for researchers working on falls prevention.

\section{Acknowledgements}

The authors would like to thank all the volunteers who participated in the study, and other researches for their contribution to the study: Aitor Belloso, Ainara Garzo (Fatronik-Tecnalia, San Sebastian, Spain) and Letitia Coello (Ingema Foundation, San Sebastian, 
Spain). The authors would also like to thank FatronikTecnalia for providing the facilities necessary to the experimental data collection.

\section{References}

[1] Y. Breniere Why we walk the way we do? Journal of Motor Behavior 28 (1996), 291-298.

[2] O. Caron, B. Faure and Y. Breniere, Estimating the center of gravity of the body on the basis of the center of pressure in standing posture, Journal of Biomechanics 30 (1997), 11691171.

[3] G.L. Cavalheiro, M.F.S. Almeida, A.A. Pereira and A.O. Andrade, Study of age-related changes in postural control during quiet standing through linear discriminant analysis, Biomedical Engineering Online 8 (2009), 35. PMID: 19922638

[4] Z. Cook, S. Kirk, S. Lawrenson and S. Sandford, Use of BMI in the assessment of undernutrition in older subjects: reflecting on practice, The Annual Meeting of the Nutrition Society 64(3) (2005), 313-317.

[5] S. Cotton, A.P. Murray and P. Fraisse, Estimation of the center of mass using statically equivalent serial chain modeling, in ASME International Design Engineering Technical Conferences, San Diego, California, USA, 2009a.

[6] S. Cotton, A.P. Murray and P. Fraisse, Estimation of the center of mass: from humanoid robots to human beings, IEEE/ASME Transactions on Mechatronics 14(6) (2009b), 707-712.

[7] P. DeLeva, Adjustments to Zatsiorsky-Seluyanov's segment inertia parameters, J Biomech 29(9) (1996), 1223-1230.

[8] W.T. Dempster, O.D. Schipplein, G.B.J. Andersson, T.P. Andriacchi, properties of body segments based on size and weight, Am J Anat 120(1) (1967), 33-54.

[9] W.R. Frontera, V.A. Hughes, K.J. Lutz and W. J. Evans, A cross-sectional study of muscle strength and mass in 45- to 78-yr-old men and women, J Appl Physiol 71(2) (1991), 644650.

[10] M. Galli, M. Crivellini, F. Sibella, A. Montesano, P. Bertocco and C. Parisio, Sit-to-stand movement analysis in obese subjects, International Journal of Obesity and Related Metabolic Disorders: Journal of the International Association for the Study of Obesity 24(11) (2000), 1488-1492. PMID: 11126346

[11] K. Hellstrom, B. Vahlberg, C. Urell and M. Emtner, Fear of falling, fall-related self-efficacy, anxiety and depression in individuals with chronic obstructive pulmonary disease, Clinical Rehabilitation 23(12) (2009), 1136-1144. PMID: 19906765

[12] N. Kanekar, M.J. Santos and A.S. Aruin, Anticipatory postural control following fatigue of postural and focal muscles, Clinical Neurophysiology: Official Journal of the International Federation of Clinical Neurophysiology 119(10) (2008), 2304-2313. PMID: 18752990
[13] D. King and V.Zatiorsky, Extracting gravity line displacement from stabilographic recordings, Journal of Gait and Posture 6 (1997), 27-38

[14] U. Lindemann, R. Muche, M. Stuber, W. Zijlstra, K. Hauer and $\mathrm{C}$. Becker, Coordination of strength exertion during the chair-rise movement in very old people, The Journals of Gerontology. Series A, Biological Sciences and Medical Sciences 62(6) (2007), 636-640. PMID: 17595420

[15] S.J. Manoogian, D.M. Moorcroft and S.M. Duma, Evaluation of pregnant female injury risk during everyday activities, Biomedical Sciences Instrumentation 44 (2008), 183-188. PMID: 19141913

[16] M.P. Murray, A. Seireg and R.C. Scholz, Center of gravity, center of pressure, and supportive forces during human activities, Journal of Applied Physiology 23(6) (1967), 831-838. PMID: 6065053

[17] J.A. Painter, S.J. Elliott and S. Hudson, Falls in communitydwelling adults aged 50 years and older: prevalence and contributing factors, Journal of Allied Health 38(4) (2009), 201-207. PMID: 20011818

[18] V.P. Panzer, S. Bandinelli and M. Hallett, Biomechanical assessment of quiet standing and changes associated with aging, Archives of Physical Medicine and Rehabilitation 76(2), (1995), 151-157. PMID: 7848073

[19] K. Schneider and R.F. Zernicke, Mass, center of mass, and moment of inertia estimates for infant limb segments, Journal of Biomechanics 25(2) (1992), 145-148. PMID: 1733990

[20] T. Shimba, An estimation of center of gravity from force platform data, Journal of Biomechanics, 17 (1984), 53-60.

[21] H. Slijper and M. L. Latash, The effects of muscle vibration on anticipatory postural adjustments, Brain Research 1015(1-2) (2004), 57-72. PMID: 15223367

[22] Z. Taha, I.M. Jomoah and H.R. Zadry, A study of anthropometric characteristics between Malaysian and Saudi Arabian males aged 20 to 30 years, Journal of Human Ergology 38(1) (2009), 27-32. PMID: 20034316

[23] D.G. Thelen, Adjustments of muscle mechanics model parameters to simulate dynamic contractions in older adults, $J$ Biomech Eng - Transactions of the ASME 125 (2003), 70-77.

[24] L.V. Thompson and M. Brown, Age-related changes in contractile properties of single skeletal fibers from the soleus muscle, J Appl Physiol 86(3) (1999), 881-986.

[25] A.A. Vandervoot and A.J. McComas, Contractile changes in opposing muscle of the human ankle joint with aging, $J$ Appl Physiol 61(1) (1986), 361-367.

[26] D.A. Winter, Biomechanics of Human Movement, Wiley, New York, 2005.

[27] V. Zatsiorsky and V. Seluyanov, The mass and inertia characteristics of the main segments of the human body, in Int Series Biomechanics, Vol. 4B, Biomechanics VIII-B, H. Matsui and K. Kobayashi eds. Vol. VIII(B) Human Kinetics 4B, Champaign, IL, pp. 1152-1159. 

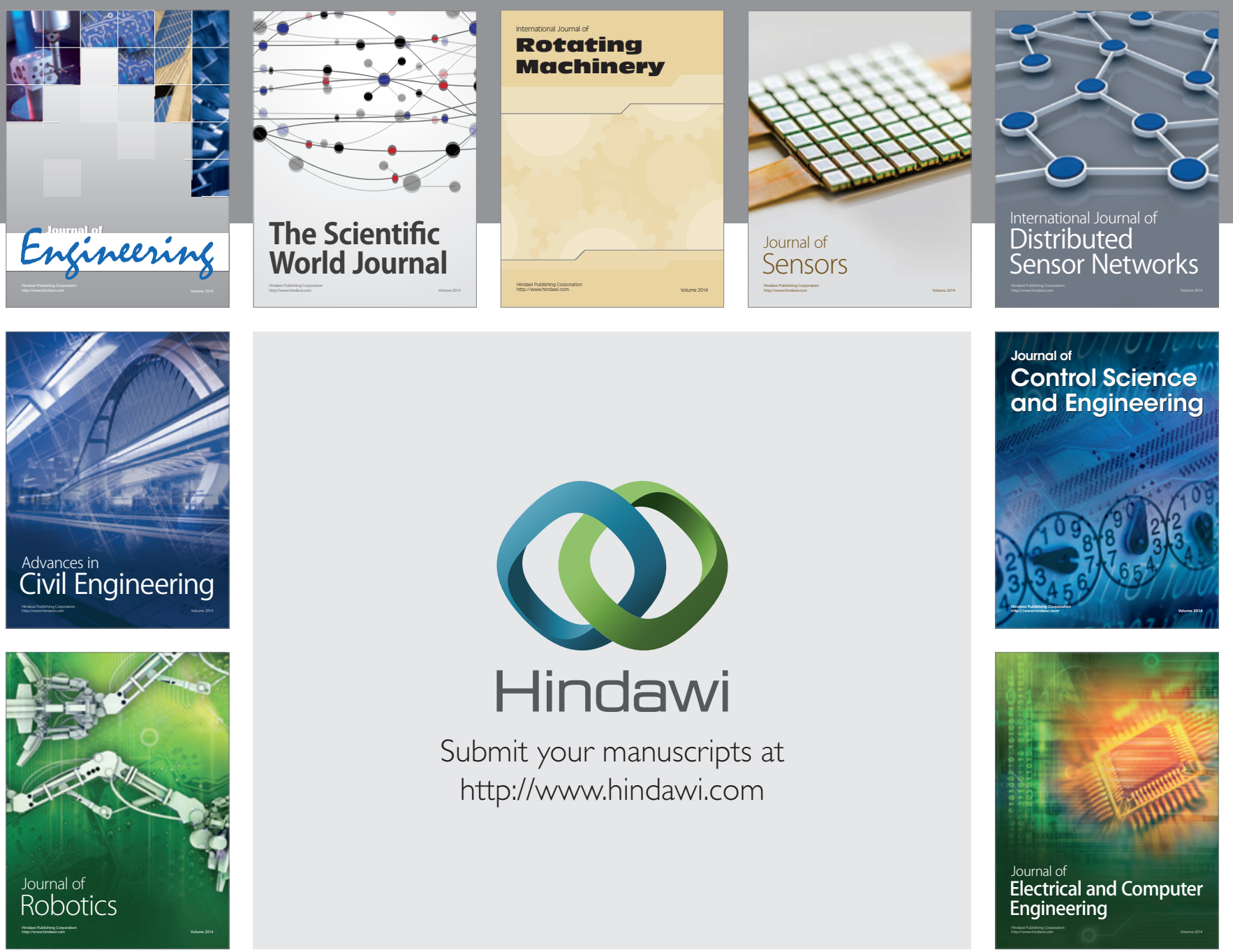

Submit your manuscripts at

http://www.hindawi.com
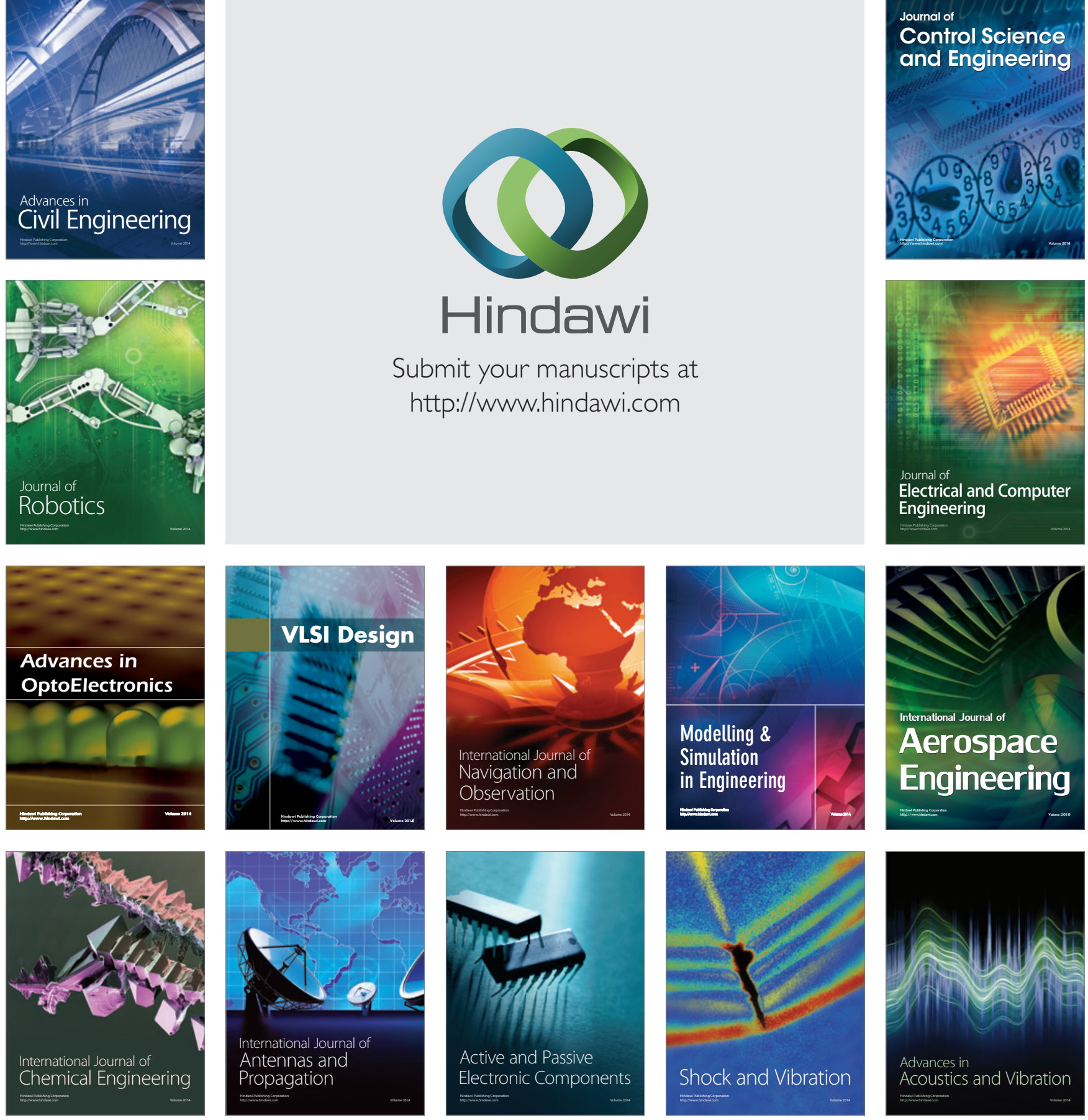